\title{
Thermal Expansion of Free-Volume in Hexadecane Confined in the Silica Gel Nanopores
}

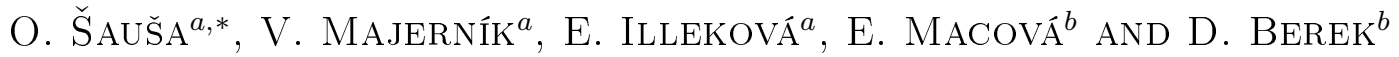 \\ ${ }^{a}$ Institute of Physics, Slovak Academy of Sciences, Dúbravská cesta 9, SK-845 11 Bratislava, Slovakia \\ ${ }^{b}$ Polymer Institute, Slovak Academy of Sciences, Dúbravská cesta 9, SK-845 41 Bratislava, Slovakia \\ Temperature dependence of the free-volume of voids in hexadecane (HXD) confined in the $3 \mathrm{~nm}$ silica gel \\ pores (Develosil-30) was measured by positron annihilation lifetime spectroscopy. Different amount of HXD was \\ filled into the pores of matrix. The anomalous thermal expansion was observed in the partially filled pores with \\ extremely low filling. The thermal expansion coefficient of free-volume voids in this solidified HXD layer has a \\ negative sign at temperatures below $180 \mathrm{~K}$ compared to high- or full-filled pores of silica gel. At these temperatures, \\ the positron annihilation lifetime spectroscopy measurements demonstrate the appearance of a new type of the \\ free volume within HXD which has been interpreted as the cracks in the HXD layer on the inner walls of matrix \\ cavities. The differential scanning calorimetry method confirmed that the processes were enacted within pores \\ and not on the outer surfaces of silica gel grains. In the sample with the extremely low filling, only the one HXD \\ solidification/melting effect connected with confined states is manifested.
}

DOI: 10.12693/APhysPolA.125.798

PACS: 68.60.-p, 78.67.Rb, 78.70.Bj

\section{Introduction}

In our previous study of confined molecules of hexadecane (HXD) in partially filled pores of controlled pore glass $(\mathrm{CPG})$ we found some basic lifetime characteristics at room temperature only [1]. The aim of this present work was to study temperature dependence of free volume of HXD confined in nanopores of silica gel (SG) at different saturations of matrix pores. The temperature dependence of the free volume of voids of HXD $V_{h}$ in the fully filled pores of SG [2] showed their continuous decreasing with decreasing temperature. In the samples with overfilled pores, differential scanning calorimetry (DSC) measurements indicated two crystallization peaks (one for confined state with the position dependent on the pore size, $R_{c}$ and one for the bulk state with a stable position in temperature range, $R_{b}$ ) [3]. In this paper, the question of the reason for the existence of two crystallization peaks, $R_{c 1}, R_{c 2}$ [4] for the HXD confined in CPG samples and the localization of them should be answered.

\section{Experimental}

The hexadecane $\mathrm{C}_{16} \mathrm{H}_{34}$ (Lachema, Czech Republic) is a liquid at temperature above $18^{\circ} \mathrm{C}$ as its tabulated melting point is $18^{\circ} \mathrm{C}$. Its density is $0.773 \mathrm{~g} \mathrm{~mL}^{-1}$ and its molar mass is $226.45 \mathrm{~g} \mathrm{~mol}^{-1}$. The hexadecane is filled into mesoporous silica gel Develosil-30 (DEV, Nomura Chemical Japan) with the average pore size $3 \mathrm{~nm}$, surface area $700 \mathrm{~m}^{2} \mathrm{~g}^{-1}$ and pore volume $0.5 \mathrm{~mL} \mathrm{~g}^{-1}$. After the water elimination, these pores were filled by a defined

*corresponding author; e-mail: ondrej.sausa@savba.sk amount of HXD at ambient temperature [3]. The actual amount of HXD was controlled by weighting with the precision of $\pm 0.01 \mathrm{wt} \%$. The amount of HXD in DEV is characterized by the coefficient of filling $k$ defined as $k=m_{\mathrm{HXD}} /\left(m_{\mathrm{DEV}}+m_{\mathrm{HXD}}\right)$, where $m_{\mathrm{HXD}}$ and $m_{\mathrm{DEV}}$ is the mass of HXD and DEV in the sample, respectively. The set of samples with $k=0.294,0.202,0.105$, and 0.05 was prepared and used for this work.

Positron annihilation lifetime spectra were measured by the conventional fast-fast coincidence method using plastic scintillators. The time resolution (FWHM) was about 320 ps and it was measured by the defect free $\mathrm{Al}$ sample. Analyses of lifetime spectra were processed by the LT program [5]. The longest-lived components were ascribed to the decay of ortho-positronium (o-Ps). At selected temperatures, the lifetime distributions were also computed by the program MELT [6] from the measured lifetime spectra.

The low temperature measurements in the temperature region from 15 to $300 \mathrm{~K}$ were made by the He closed-cycle refrigerator Leybold RD 210. The stability of the temperature was about $1 \mathrm{~K}$. The samples were kept in vacuum of about $2 \mathrm{~Pa}$.

Power-compensation DSC8500 (Perkin-Elmer) with automatic intracooler $(200 \mathrm{~K})$, encapsulated samples of 15 to $30 \mathrm{mg}$ and argon atmosphere was used. Continuous cooling and heating regimes with the rates of $\beta= \pm 2 \mathrm{~K} \mathrm{~min}^{-1}$ were applied. The precision of measured data is $\pm 0.4 \mathrm{~K}$ and $\pm 2 \mathrm{~J} \mathrm{~g}^{-1}$.

\section{Results and discussion}

Figure 1a shows the $\tau_{3}$ lifetime as well as the average free-volume of voids $\left(\mathrm{V}_{\mathrm{h}}\right)$ as a function of temperature in HXD confined in DEV. $\mathrm{V}_{\mathrm{h}}$ were computed from $\tau_{3}$ lifetime by commonly employed Tao-Eldrup formula $[7,8]$. 
Four-component analysis was used in this case. In the samples with coefficient of filling $k<0.2$, the temperature dependence of $\mathrm{V}_{\mathrm{h}}(T)$ is changing and it starts to increase with decreasing temperature below $180 \mathrm{~K}$. This $k$ corresponds to about $65 \%$ pore saturation for DEV as estimated from the pore volume given by producer and the weight of HXD added. Such anomalous behaviour of $\mathrm{V}_{\mathrm{h}}(T)$ is more visible for decreased coefficient of filling, $k$. The intensity $I_{3}$ of this $o$-Ps component is in Fig. $1 \mathrm{~b}$.

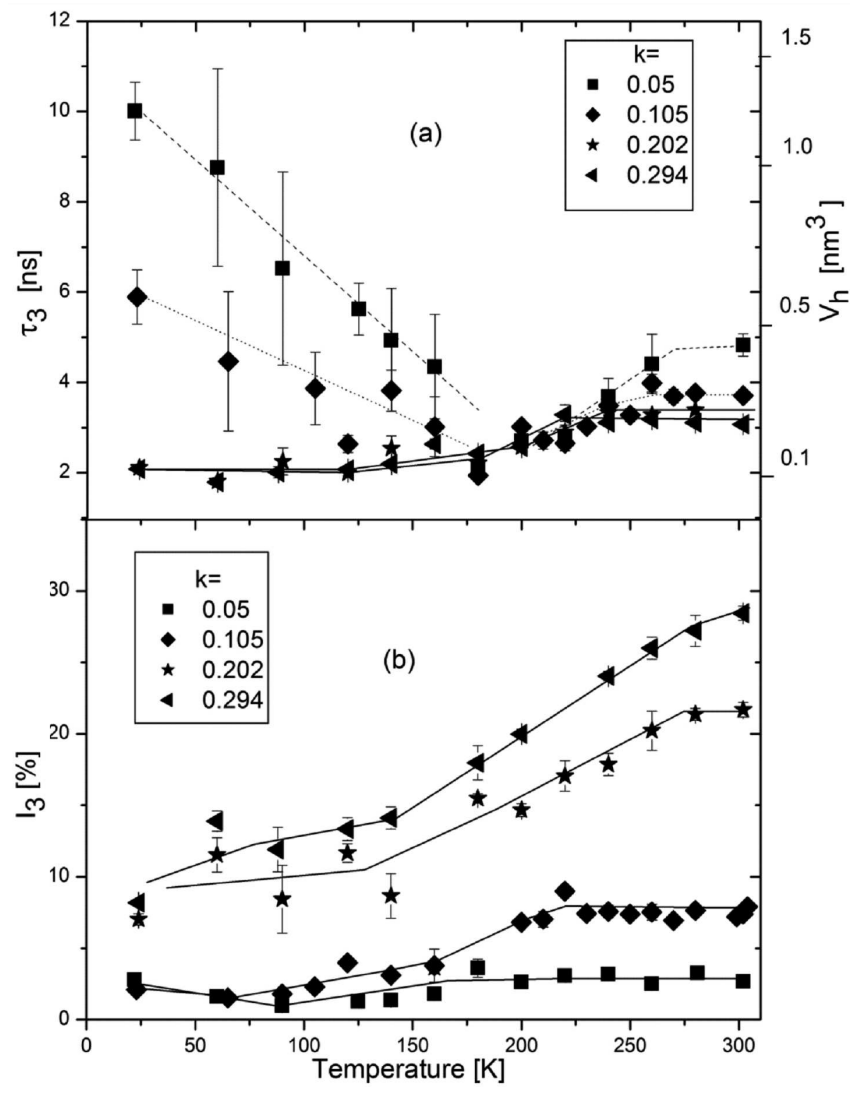

Fig. 1. (a) Average free-volume of voids $V_{h}$ as a function of temperature in HXD confined in DEV as well as $\tau_{3}$ lifetime for various $k$ and (b) corresponding relative intensity $I_{3}$.

Figure $2 \mathrm{a}$ and $\mathrm{b}$ shows the lifetime distributions (1-10 ns range) obtained by MELT for $k=0.105$ and 0.202 (pore saturation $30 \%$ and $65 \%$, respectively) and for different temperatures. The anomalous effect for the $k=0.105$ is visible and for the $k=0.202$ is already negligible.

The mixture of lifetime components in the range about 1-10 ns was attributed to free-volumes typical for SG (practically no temperature dependence), to solid HXD and to free-volumes between HXD and surface of the SG matrix, similar to [9]. Last two free-volumes are temperature dependent and we suppose that the shorter lifetime (1-4 ns) can be attributed to the free-volume of solid HXD according to our last temperature measurements on fully filled samples [2]. Then the anomalous thermal

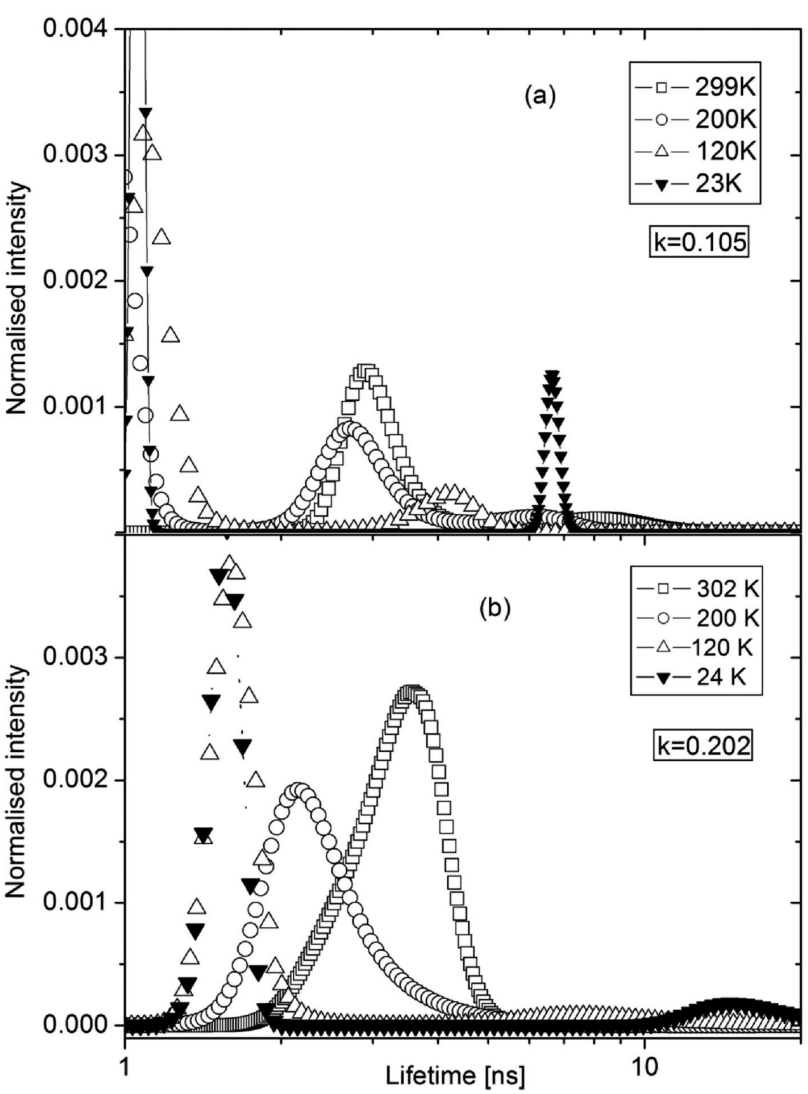

Fig. 2. Lifetime distributions for shorter $o$-Ps lifetimes as a function of temperature for different extent of filling expressed by $k$. (a) 0.105 and (b) 0.202 .

expansion in Fig. 1 may be explained by the cracking of the thin layer of HXD at the surface of DEV pores. No significant changes of apparent dimensions of pores with large diameters $\left(\tau_{4}\right)$ for the $k=0.05,0.105$, and 0.202 were observed in this DEV matrix (Fig. 3a). Average value of the longest $o$-Ps lifetime is $\approx 70 \mathrm{~ns}$ for vacuum and $300 \mathrm{~K}$. It indicates that HXD does not create the homogeneous layer on the internal surface of all pores, compared for instance with HXD in $12.6 \mathrm{~nm}$ pores of CPG and $k \geq 0.3$ [1], in which the diameter of large pores goes slowly down with increase of $k$. The relative intensity of the long lifetime component depends on the quantity of HXD in DEV (Fig. 3b). For the $k=0.294$ (110.2\% pore saturation, overfilled sample) no long lifetime component was observed.

DSC cooling and heating curves for samples with various $k$ and also DSC curves for pure HXD are presented in Fig. 4. DSC measurements show one or two (for the fully filled sample) peaks $R_{c}$ and $R_{b}$ for melting or solidification of HXD in DEV. It is known that HXD flowing into nanopores of the confining matrix first wets the pore surface and only afterwards it fills the interior [4]. For this reason, we decreased the content of HXD in samples (various fillings). Then, the lower temperature solidification peak splitted into two sub peaks $R_{c 1}$ and $R_{c 2}$. In case of 


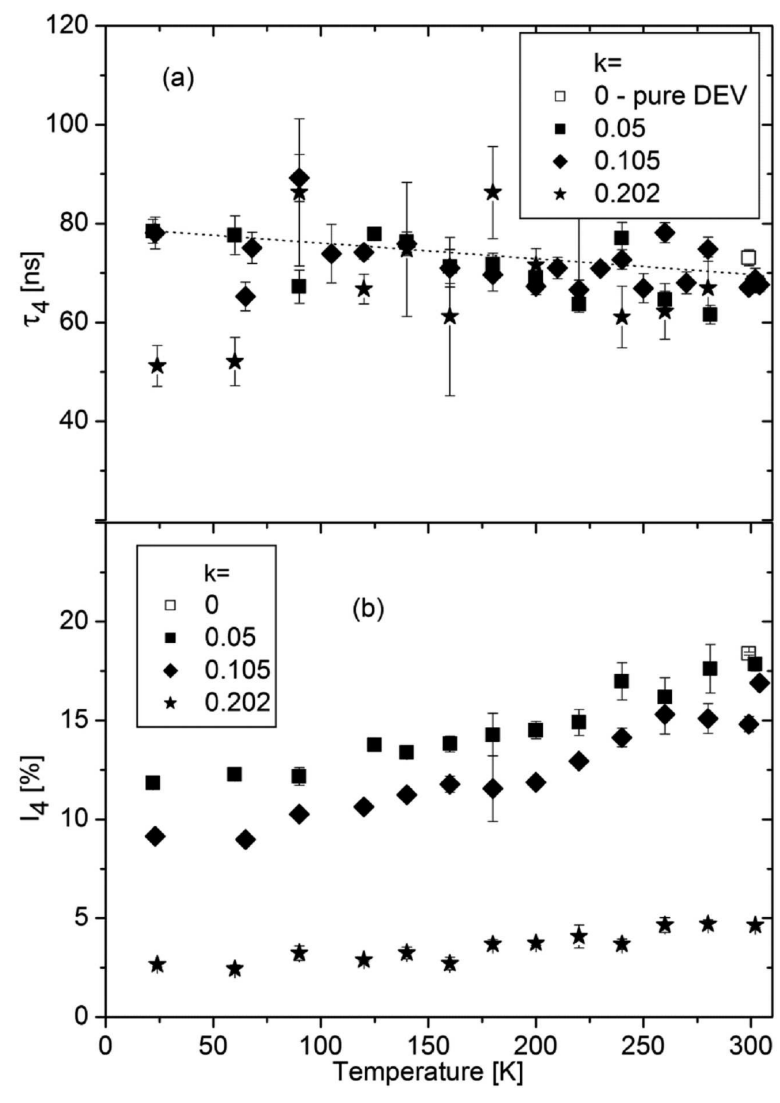

Fig. 3. Temperature dependence of the longest lifetime component $\tau_{4}$ (a) and of the corresponding intensity $I_{4}$ (b) for various $k$ of HXD in DEV. The point for the pure $\operatorname{DEV}(k=0)$ is added for comparison.

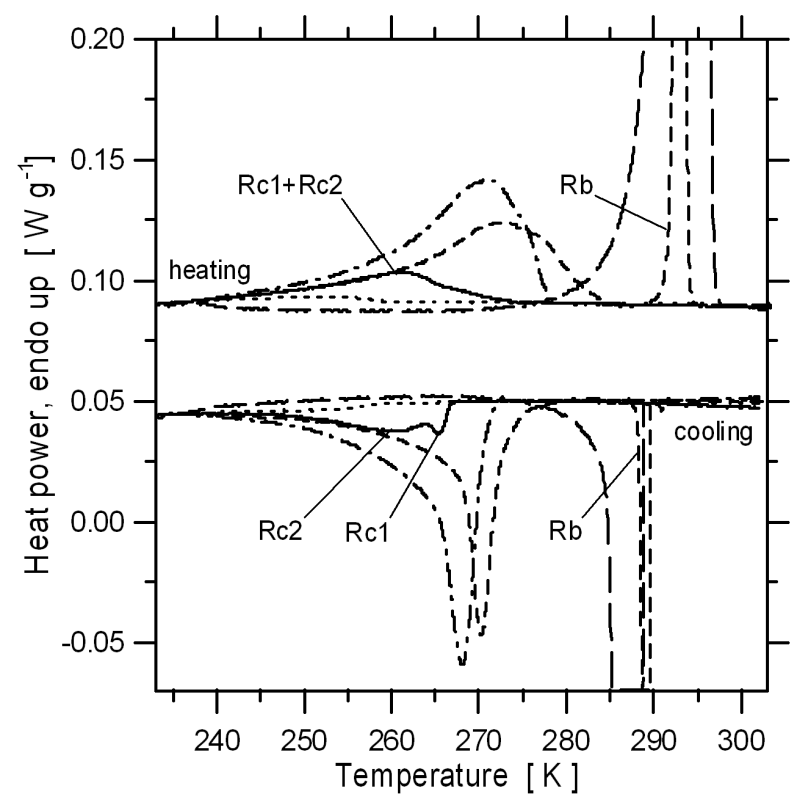

Fig. 4. Cooling and heating DSC curves for HXD confined in DEV with various coefficients of filling $k$ $(0.05 \cdots, 0.105-, 0.202-\cdots,-, 0.294-\cdots)$. Also DSC curves for pure HXD (_- - - ) are presented.
CPG with larger pores ( $>6 \mathrm{~nm},[9])$ both $R_{c 1}$ and $R_{c 2}$ peaks are visible in the cooling cycle, but for DEV with smaller pores ( $3 \mathrm{~nm}$, length of HXD molecules is $2.2 \mathrm{~nm}$ ) these $R_{c 1}$ and $R_{c 2}$ peaks are seen only for $k=0.104$. For $k=0.05$ (Fig. 4 ) the small $R_{c 2}$ peak was seen only probably. This single peak $R_{c 2}$ is assigned to the presence of the "thin interfacial layer" of confined HXD. For other values of $k$, the summary $R_{c 1}+R_{c 2}$ effects is observed.

\section{Conclusions}

Anomalous behaviour of the dependence $\mathrm{V}_{\mathrm{h}}(T)$ below $180 \mathrm{~K}$ for hexadecane in the partially filled pores of silica gel $(k<0.2)$ is observed. It is interpreted by the cracking of the thin layer of HXD at the surface of DEV pores.

No significant changes of large pore diameters for different amounts of HXD (for $k<0.294$ ) were observed. This component does not exist for samples with $k=$ 0.294 (fully filled pores by HXD).

DSC measurements show one (for the partially filled) and two (for the overfilled sample) peaks $R_{c}$ and $R_{b}$ for melting or solidification of $\mathrm{HXD}$ in SG. $R_{c 1}$ and $R_{c 2}$ peaks are seen for $k=0.104$ and only the $R_{c 2}$ peak is seen for $k=0.05$. For the larger $k$ the summary $R_{c 1}+R_{c 2}$ effects is observed and for overfilled sample with $k=0.294$ the $R_{b}$ peak is also present.

Results shown and discussed in this work are important for better understanding of phase transitions, adsorption and properties of materials in confined geometries.

\section{Acknowledgments}

Support of the Agency of the Ministry of Education of the SR for the Structural Funds of the EU (Cekomat II, ITMS 26240120020) is gratefully acknowledged. The authors wish to thank the grant agency VEGA for support by the grant No. 2/0111/11, 2/0171/09, 2/0164/14 as well as grant agency APVV for the support by the grant No. 0647-10 and 0109-10.

\section{References}

[1] O. Šauša, E. Illeková, J. Krištiak, D. Berek, E. Macová, J. Phys., Conf. Series 443, 012059 (2013).

[2] M. Iskrová, V. Majerník, E. Illeková, O. Šauša, D. Berek, J. Krištiak, Mater. Sci. Forum 607, 235 (2009).

[3] E. Illeková, M. Miklošovičová, O. Šauša, D. Berek, J. Therm. Anal. Calorim. 108, 497 (2012).

[4] E. Illeková, J. Krištiak, E. Macová, I. Mat'ko, O. Šauša, J. Therm. Anal. Calorim. 113, 1187 (2013).

[5] J. Kansy, Nucl. Instrum. Methods Phys. Res. A 374, 235 (1986).

[6] A. Shukla, M. Peter, L. Hoffman, Nucl. Instrum. Methods Phys. Res. A 335, 310 (1993).

[7] S.J. Tao, J. Chem. Phys. 56, 5499 (1972).

[8] M. Eldrup, D. Lightbody, J.N. Sherwood, Chem. Phys. 63, 51 (1981)

[9] R. Zaleski, W. Dolecki, A. Kierys, J. Goworek, Micropor. Mesopor. Mater. 154, 142 (2012). 